\title{
Comparative Performance Study of Strain Gauge Sensors for Ion Thrust Measurement
}

$\begin{array}{ll}\text { R. John Stephen } & \text { Non-member } \\ \text { K. Rajanna* } & \text { Non-member } \\ \text { Vivek Dhar }^{* *} & \text { Non-member } \\ \text { K.G.Kalyan Kumar }^{* *} & \text { Non-member } \\ \text { S.Nagabushanam }^{* *} & \text { Non-member }\end{array}$

A strain gauge based thrust measurement system has been developed, by fabricating a thrust balance with four columns. In order to compare the performance of the different types of strain gauges for ion thrust measurement, foil type strain gauges were bonded to the first column and semiconductor strain gauges were bonded to the second column. Whereas, thin film strain gauges of $\mathrm{NiCr}$ and $\mathrm{Pt}-\mathrm{W}$ materials were deposited on to the third and fourth columns respectively. The strain gauges of a particular type in each column, connected in Wheatstone bridge configuration, were calibrated to measure the thrust produced using Lami's principle and the performance was studied. Strain gauge bridge sensitivities varied from about $6 \mu \mathrm{V} / \mathrm{mN}$ to $115 \mu \mathrm{V} / \mathrm{mN}$ and the non-linearity varied from about $0.98 \%$ to $2.84 \%$ for bridges formed by the different types of strain gauges. Details on the calibration and simultaneous comparative performance study of the system developed have been presented

Keywords: strain gauge sensors, thrust balance, ion thrust.

\section{Introduction}

Strain gauge is a device or sensor used to measure the linear deformation occurring in the material during loading. These sensors are used for a variety of applications such as measurement of load, force, pressure, etc. Of the various kinds of applications, thrust measurement plays an important role in space applications where electric propulsion thrusters are mounted on the satellites and activated for reasons such as orbit maintenance, re-positioning and attitude control ${ }^{(1)-(4)}$. In our earlier papers, we have reported the use of conventional foil type of strain gauges ${ }^{(5)}$ and thin film strain gauge sensors ${ }^{(6)}$ for the measurement of thrust produced by Hall thrusters such as Stationary Plasma Thruster (SPT).

In the present paper, we report the details of the calibration and comparative performance study of the thrust measurement system developed which includes a thrust balance assembly with different types of strain gauge sensors such as foil type, semiconductor type and thin film strain gauge sensors made of NiCr and Pt-W materials with associated signal conditioning circuitry. Thus, a simultaneous comparative performance study of the various types of strain gauge sensors for ion thrust measurement has been carried out and the results obtained are presented.

\footnotetext{
* Department of Instrumentation, Indian Institute of Science, Bangalore 560 012, INDIA.

** Control Systems Group, ISRO Satellite Center,

Bangalore 560 017, INDIA.
}

\section{Types of Strain Gauges}

Strain gauges based on electrical, mechanical, optical, acoustical and pneumatic principles are known ${ }^{(7)}$. Of these the electrical strain gauges have become so widely accepted that they now dominate the entire strain gauge field. In its widest sense, the electrical strain gauge includes many varieties such as resistance, capacitance and inductance. However, over the years, the electrical resistance strain gauge has become the most widely used device, and this is what is usually meant when the term strain gauge is used. The popular types of strain gauges belonging to this category are metallic foil type, semiconductor type and thin film strain gauges. Foil type strain gauge sensor is the most common one; semiconductor type of strain gauge is known for its high sensitivity. A major advantage of thin film strain gauges is that, in the case of conventional strain gauges the resistive foil attached to a thin phenolic/epoxy sheet has to be bonded to the diaphragm or specimen undergoing the strain using a suitable adhesive. Since the adhesive and the backing material are in the force path, the accuracy of measurement is limited by the characteristics of these materials ${ }^{(8)}{ }^{(9)}$. As thin film strain gauges can be directly deposited (with a dielectric film for insulation) on the diaphragm or the specimen, the limitation of accuracy due to phenolic/epoxy material is totally eliminated. 


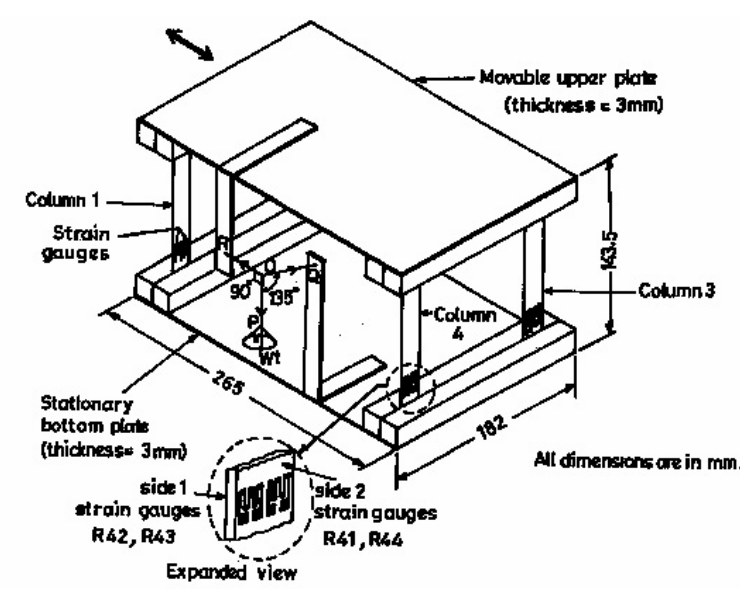

Fig. 1. Schematic of the thrust balance assembly

\section{Thrust Balance Assembly}

The thrust measurement system was developed by fabricating a thrust balance assembly. Fig. 1 shows the schematic of the thrust balance assembly. It consists of a stainless steel (SS) base plate (or bottom plate) with four columns of beryllium-copper, mounted one on each of the four corners, simply supporting another stainless steel plate at the top. The bottom plate is stationary and the upper plate moves in response to a force while remaining parallel to the bottom plate. The critical load $\mathrm{P}$, which is the minimum load required to make the columns buckle was determined using relation (1),

$$
P=\frac{4 \pi^{2} E I}{L^{2}} \ldots \ldots \ldots \ldots \ldots \ldots \ldots \ldots \ldots
$$

where $\mathrm{E}$ is the Youngs modulus of the material of the column, I is the moment of inertia and L is the length of the column. The critical load for our present system has been determined to be $3.8 \mathrm{~kg}$. When the thrust balance is critically loaded, even a small force in the transverse direction produces a large deflection of the columns, the deflection being proportional to the magnitude of force acting on them. While carrying out tests on the thrust measuring system, the ion thruster of mass $0.7 \mathrm{~kg}$ mounted on a stand was placed on the top plate of the thrust balance. Additional weights in the form of circular SS disks were attached to the bottom of the upper SS plate so as to critically load the thrust balance. The thruster and the weights were placed at the center of the upper plate in order that the four columns share the total load equally. To provide damping during the operation of the thruster, a glass beaker filled with viscous oil was placed on the bottom SS plate. A rectangularly shaped aluminium plate, partially immersed in the oil was connected to the upper SS plate so as to form a viscous damper during the motion of the balance.

\section{Installation/Deposition of Strain Gauges}

Strain gauges had to be bonded to or deposited on the region of the four columns of the thrust balance, where the strain is maximum. This region was found to be at a distance of approximately $30 \%$ of the length of the column from its either end. The region from the lower end was preferred for bonding or deposition of the gauges because of better accessibility during wiring. Of the four columns, the first column was bonded with foil type of strain gauges of $\mathrm{NiCr}$ material. Two such gauges were bonded on either side of this column using the standard procedure recommended by the manufacturer. Commercially available strain gauges of semiconductor material were bonded on to the second column. In comparison with foil type of strain gauges, thin film strain gauges offer several advantages. Hence, thin film strain gauges of $\mathrm{NiCr}$ material were deposited on the third column so that a comparative study can be made with that of foil type strain gauges. Similarly, thin film strain gauges of platinum-tungsten (Pt-W) material were deposited on the fourth column. The four strain gauges in each column were connected in wheatstone bridge configuration such that the two gauges on one side of the column (which undergo tensile strain) form one pair of opposite arms of the wheatstone bridge and the remaining two gauges on the other side of the column (which undergo compressive strain) form the other pair of opposite arms of the bridge. This arrangement produces maximum differential output voltage for a given deflection of the columns. In addition, it also offers the advantage of adequate temperature compensation ${ }^{(10)}$. The details of the preparation and deposition of thin film strain gauges are provided in our earlier publication ${ }^{(6)}$.

4.1 Determination of Gauge Factor It is essential to know the sensitivity or the gauge factor of the strain gauges to assess their suitability for practical use. For the foil type of strain gauges the manufacturer provides the value of gauge factor. However, for the other strain gauges such as the thin film strain gauges, this value is to be experimentally found.

In order to determine the gauge factor of the thin film strain gauges deposited, a mechanical setup was designed and developed. The set up consists of a rectangular base plate made of stainless steel material with a cylindrical rod fixed vertically at the left wherein a fixture has been provided for holding the strain gauge columns in the form of a cantilever. The free end of the cantilever can be deflected by means of screw gauge (micrometer) fixed to a holder on the dovetail arrangement, which can be made to move along the length of the base plate. Fig. 2 shows the photograph of the cantilever set up with the electronic accessories and the necessary signal conditioning circuitry. The free end of the cantilever was deflected in steps and the corresponding bridge output voltages were observed for the columns with various types of strain gauges and were found to be linear.

Fig. 3 shows the variation of cantilever deflection versus bridge output for $\mathrm{NiCr}$ thin film strain gauges for different excitation voltages. Fig. 4 shows the variation of deflection of cantilever versus bridge output for the column with semiconductor strain gauges, for increasing and decreasing deflections. 


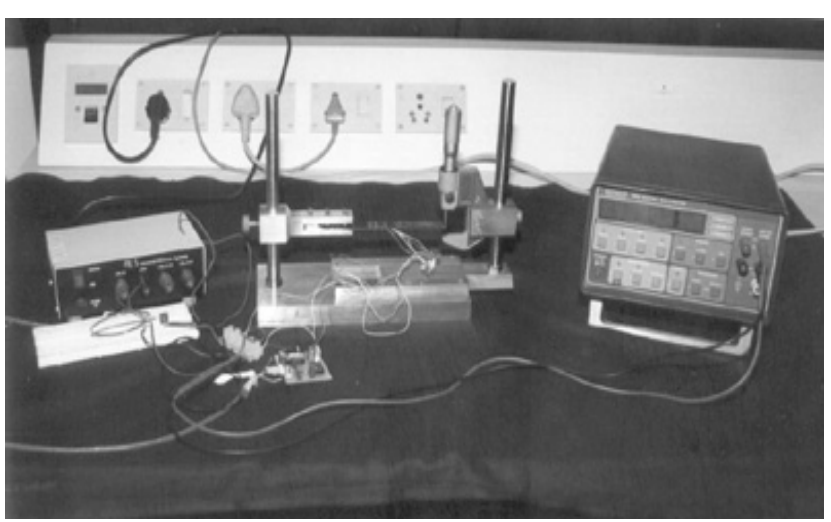

Fig. 2. Photograph of the cantilever setup with accessories

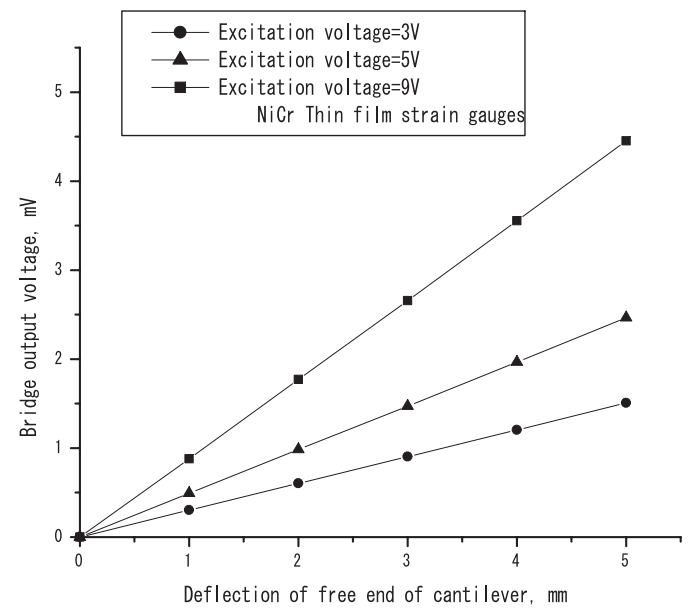

Fig. 3. Deflection of cantilever vs. bridge output for $\mathrm{NiCr}$ thin film strain gauges

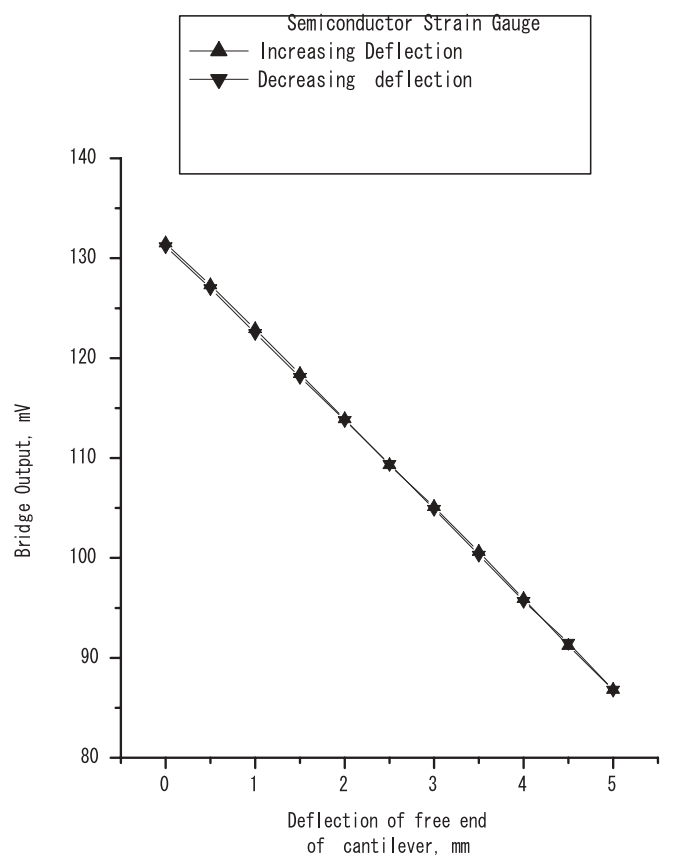

Fig. 4. Deflection of cantilever vs. bridge output for semiconductor strain gauges

\section{Method of Calibration and Perfor- mance Study}

In order to calibrate the thrust measurement system, an arrangement was made in the thrust balance using a fine silk thread with a pan as shown in Fig. 1. The thread was tied such that the plane PQR formed by the thread was parallel to the vertical plane of the thrust balance. A mild steel nut of mass $1.03 \mathrm{~g}$ was attached to point $\mathrm{P}$ of the thread, whereas points $\mathrm{Q}$ and $\mathrm{R}$ were tied to the thrust balance. The thread OR was parallel to the horizontal plane and the thread OP was hanging vertically downward due to the weight attached to its lower end. Thus $\angle P O R=90^{\circ}$. Point $\mathrm{Q}$ of the thread was tied to the thrust balance such that $\angle P O Q=\angle R O Q=135^{\circ}$.

If $F_{P}$ is the force acting along OP and $F_{Q}$ the force acting along $\mathrm{OQ}$ and $F_{R}$ the force acting along OR, according to Lami's theorem,

$$
\begin{aligned}
& \frac{F_{P}}{\sin \angle R O Q}=\frac{F_{R}}{\sin \angle P O Q}=\frac{F_{Q}}{\sin \angle P O R} \\
& \frac{F_{P}}{\sin 135^{\circ}}=\frac{F_{R}}{\sin 135^{\circ}}=\frac{F_{Q}}{\sin 90^{\circ}}
\end{aligned}
$$

Therefore,

$$
F_{P}=F_{R}
$$

The downward force $F_{P}$ due to a mass of $1.03 \mathrm{~g}$ acting along OP is equal to the product of mass and the acceleration due to gravity, which is $10.1 \mathrm{mN}$. Hence the force $F_{R}$ acting along OR is also equal to $10.1 \mathrm{mN}$.

The vacuum chamber used for the purpose of measurement is a cylindrical chamber of $1.2 \mathrm{~m}$ diameter and $1.4 \mathrm{~m}$ length made of stainless steel material. A combination of rotary, turbo molecular and cryo pumps was used to obtain pressures as low as $10^{-6}$ mbar. The rotary and the turbo molecular pumps have been mounted on rubber pads to minimize vibration and have been connected to the vacuum chamber through metallic bellows so as to reduce the transfer of vibrations to the chamber.

While performing the actual in - situ calibration, the thrust balance is placed on a horizontal platform inside the cylindrical vacuum chamber. The SPT is placed on the thrust balance and the thrust balance is critically loaded. A mass of $1.03 \mathrm{~g}$ is attached to point $\mathrm{P}$ of the thread arrangement shown in Fig. 1. A solenoid is used around the mass attached to point $\mathrm{P}$, in order to lift it whenever required. A stainless steel enclosure placed surrounding the columns of the thrust balance, protects the strain gauges from direct ion impingement on them during the operation of the thruster. The chamber is evacuated to a pressure of approximately $10^{-6} \mathrm{mbar}$ and in - situ calibration is usually done with the propellant flow $\mathrm{ON}$ at a pressure of $10^{-4}$ mbar. When the solenoid is in the normal (de-energized) state the mass hangs vertically downwards due to the force of gravity. At this condition the output voltage from the measurement system is noted. The solenoid is then energized and when the mass thus gets lifted, the output voltage is once again observed. The difference between these two voltages correspond to a force of $1.03 \times 9.8=10.1 \mathrm{mN}$. 


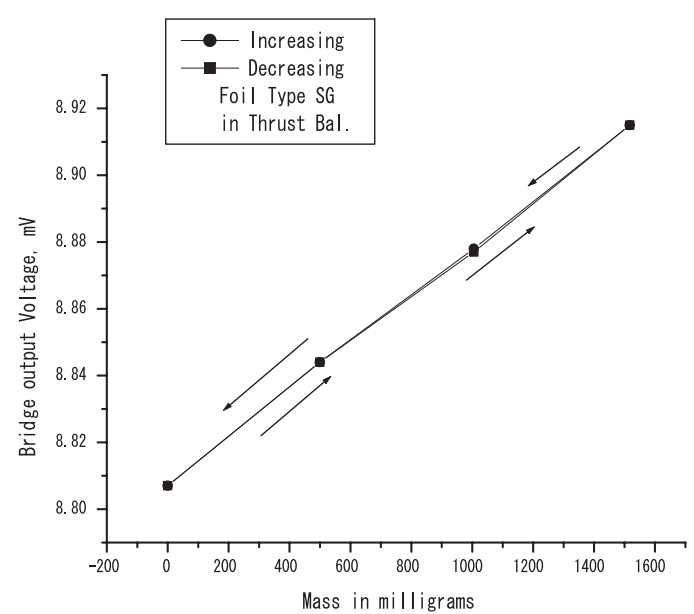

Fig. 5. Variation of mass vs. bridge output for foil type strain gauge bridge

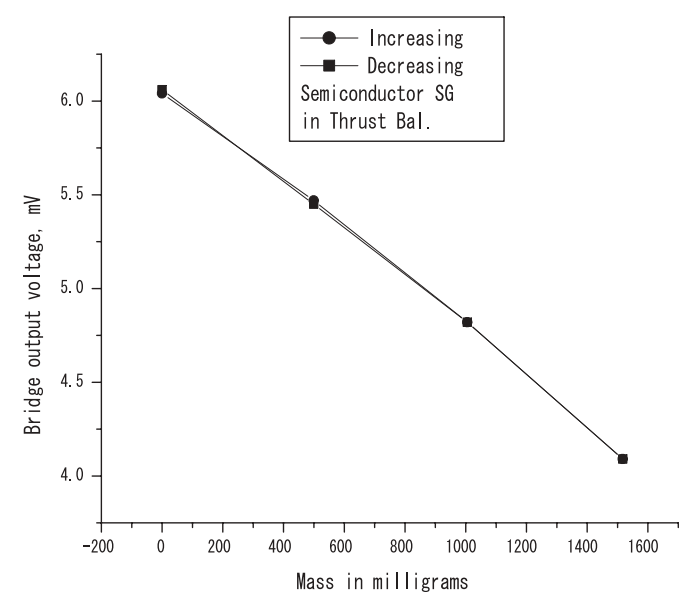

Fig. 6. Variation of mass vs. bridge output for semiconductor strain gauge bridge

The SPT is then operated and the actual change in the output voltage before and after the firing of the thruster is noted. Thus, the thrust produced by the SPT is given by,

\section{Thrust, $m N$}

$$
=\frac{\text { change in output voltage }}{\text { voltage change during insitu calibration }} \times 10.1
$$

Performance study has been carried out with the strain gauge sensors of the types mentioned in section 1 . Calibration was carried out for each type of strain gauge independently. With the thrust balance critically loaded small masses were added to the pan arrangement of the thrust balance and the bridge output voltages were measured. The variation of output voltage with mass is as shown in Figs. 5-8 for the columns with different types of strain gauges. The measured gauge factor was found to be $\sim 2.7$ for thin film strain gauge of $\mathrm{NiCr}$ material, 3.5 for the Pt-W thin film strain gauge and 39.7 for the semiconductor strain gauge. However, the gauge factor of foil type strain gauge was known to be 2.1 for $\mathrm{NiCr}$ material (data provided by the manufacturer of the strain gauge)

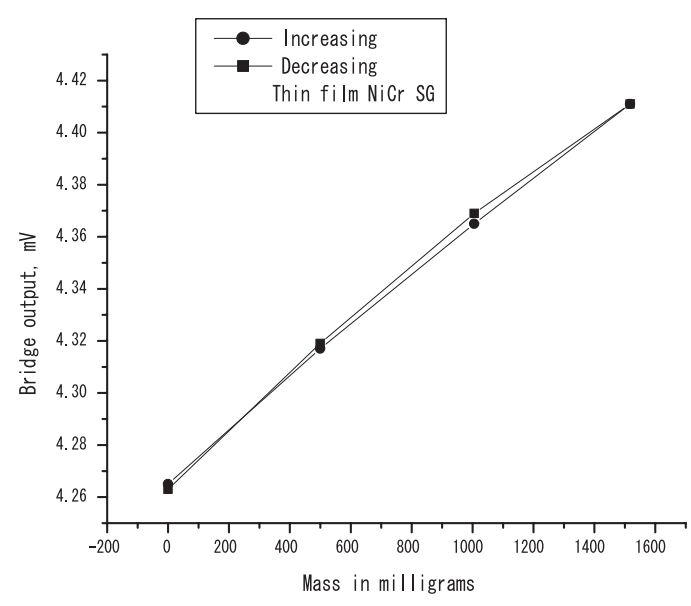

Fig. 7. Variation of mass vs. bridge output for $\mathrm{NiCr}$ thin film strain gauge bridge

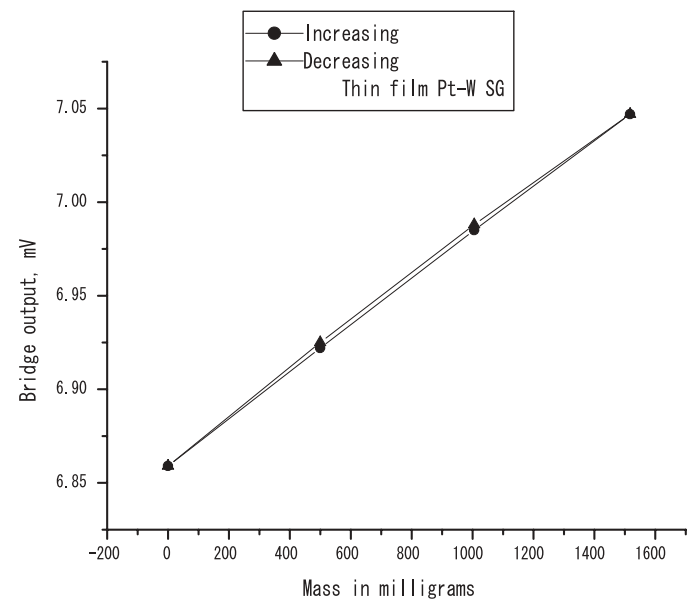

Fig. 8. Variation of mass vs. bridge output for Pt$\mathrm{W}$ thin film strain gauge bridge

Thin film strain gauge of Pt-W material showed sensitivity higher than that of conventional foil type strain gauge, and that of thin film $\mathrm{NiCr}$ gauge with good repeatability and excellent linearity. Semiconductor strain gauge showed the highest non-linearity of $2.84 \%$ although the gauge factor or sensitivity was high. Foil type strain gauges were found to have a nominal sensitivity with good repeatability. Pt-W thin film strain gauges exhibited the highest stability of all the types of strain gauges considered. Also, thin film strain gauges require greater care in handling owing to their delicacy.

Thrust measurements were done with the propellant flow ON. The measured values of thrust with the various types of strain gauge sensors were found to be in the range of $11.4-13.6 \mathrm{mN}$ for discharge powers of 220 265 watts with an accuracy of less than a milli-Newton. This variation is shown in Fig. 9. The thrust values measured at a particular discharge power with different strain gauges were found to be generally in close agreement. Semiconductor strain gauges were found to be very sensitive to temperature variations and a small drift in output was observed. 


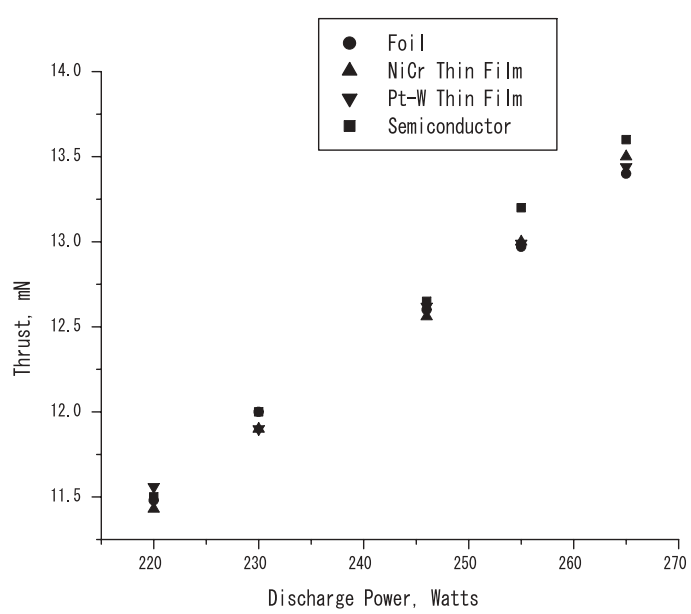

Fig. 9. Variation of thrust with discharge power for the various strain gauges

\section{Conclusion}

A simultaneous comparative study of the thrust measurement system with different types of strain gauge sensors has been performed. It has been observed that, Pt$\mathrm{W}$ thin film strain gauges exhibited good sensitivity and linearity, excellent repeatability associated with high stability. $\mathrm{NiCr}$ thin film strain gauges showed slightly lower sensitivity, good linearity and stability. Foil type strain gauges made of $\mathrm{NiCr}$ material were found to have reasonably good sensitivity, linearity and stability. In case of semiconductor strain gauges, although the sensitivity was very high, a drift in the output was noticed over a period of time.

\section{Acknowledgment}

ISRO Satellite Center (ISAC), Department of Space, has supported the work reported in this paper through a research project. The authors wish to thank Chairman, Department of Instrumentation, IISc and Group Director, Control Systems Group, ISAC for their encouragement in carrying out the work. The authors also thank all the colleagues of the respective groups in both the organizations for their help. Thanks are due to Dr.M.M. Nayak, Manager, Advanced Transducers Division, LPSC, Bangalore, for his many useful discussions and suggestions during the course of this work.

(Manuscript received Sep. 6, 2004, revised July 14, 2005)

\section{References}

(1) K. Makowski, Z. Peradzynski, N. Gascon, and M. Dudeck: "A Stationary Model for Stationary Plasma Thruster (SPT) Discharge", Proc. of 35th AIAA/ASME/SAE/SEE Joint Propulsion Conference \& Exhibit, Los Angeles, California, USA, June 20-24, AIAA pp.99-2295 (1999)

(2) L. Garrigues, A. Heron, J.C. Adam, and J.P. Boeuf: "Comparisons Between Hybrid \& PIC Models of Stationary Plasma Thruster", Proc. of 35th AIAA/ASME/SAE/ASEE, Joint Propulsion Conference \& Exhibit, Los Angeles, California, USA, June 20-24, paper AIAA-99-2297 (1999)
( 3 ) S. Roche, S. Barral, S. Bechu, M. Dudeck, P. Lasgorceix, L. Magne, T. Minea, D. Pagnon, and M. Touzeau: "Thermal Analysis of a Stationary Plasma Thruster", Proc. of 35th AIAA/ASME/SAE/SEE, Joint Propulsion Conference \& Exhibit, Los Angeles, California, USA, June 20-24, paper AIAA 99-2296.

(4) M. Day, N. Maslennikov, T. Randolph, and W. Rogers: "SPT-100 Subsystem Qualification Status", Proc. of 31st AIAA/ASME/SAE/ASEE, Joint Propulsion Conference and Exhibit, San Diego, CA, USA, July 10-12, AIAA-95-2666 (1995)

(5) R. John Stephen, K. Rajanna, Vivek Dhar, K.G. Kalyan Kumar, and S. Nagabushanam: "Strain Gauge based thrust measurement system for a stationary Plasma Thruster", Measurement Science and Technology, Vol.12, pp.1568-1575 (2001)

(6) R. John Stephen, K. Rajanna, Vivek Dhar, K.G. Kalyan Kumar, and S. Nagabushanam: "Thin film strain gauge sensors for ion thrust measurement", IEEE Sensors Journal, Vol.4, No.3, pp.373-377 (2004-6)

( 7 ) J.W. Dally and W.F. Riley: Experimental stress analysis, 2nd edition, McGraw-Hill, Kogakusha (1978)

(8) P.R. Perino: "Thin film strain gauge transducers", Instrument and Control Syst., Vol.38, p.119 (1965)

(9) K. Rajanna, S. Mohan, and E.S.R. Gopal: "Thin film strain gauges - An overview ", Indian J. pure and Appl. Physics, Vol.27, p.453 (1989)

(10) E.O. Doebelin: Measurement Systems-Applications and Design, 3rd edition, 1985, McGraw-Hill, New York, p.63 (1985)

R. John Stephen (Non-member) was born in Tiruchira-

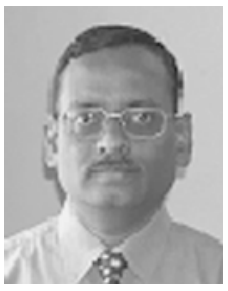
palli, Tamilnadu, India, in 1957. He received B.E. degree in Electronics/Instrumentation in the year 1982 from Annamalai University. After staying in industries for a period of about two years he joined as Lecturer, Siddaganga Institute of Technology (SIT), Tumkur, Karnataka, in the year 1984. He obtained M.Tech degree in Instrumentation Technology from Indian Institute of Science (IISc), Bangalore, India, in the year 1988. Since 1992 he has been working as Assistant Professor, Dept. of Instrumentation/Electronics, SIT, Tumkur. His fields of interest include control systems, process control and instrumentation. Presently, he is completing the requirements for the PhD degree in the Department of Instrumentation, IISc. He has publications both in National as well as in International Journals.

K. Rajanna (Non-member) was born in Karnataka State,

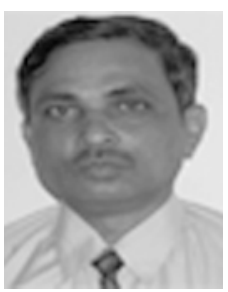
India, in 1952. He graduated from the University of Mysore, India, with a B.Sc degree in Physics and Mathematics in 1973. He received M.Sc degree in physics from the same university in 1976. He was awarded the M.Sc (Engg.) and PhD degrees from the Indian Institute of Science, Bangalore during the years 1988 and 1993 respectively. At present he is an Associate Professor in the Department of Instrumentation, Indian Institute of Science. His areas of interest include thin film sensors microsensors (MEMS), novel materials engineering for sensors applications and related instrumentation. He served as a member of the Editorial Review Committee of the Journal IEEE Transactions on Instrumentation and Measurement (USA). He was a visiting fellow of Japan Society for the Promotion of Science (JSPS) twice during 1997-1998 at the Toyohashi University of Technology, Japan. Also, he was a visiting professor at the Tohoku University, Japan during the years 2000 and 2002. 
Vivek Dhar (Non-member) obtained his B.E. Degree in Mechanical Engineering from Regional Engineering College, Allahabad, India in the year 1992 and has been working as an Engineer at the Indian Space Research Organization Satellite centre (ISAC), Bangalore, for the past eight years. Currently he is undergoing M.Tech Degree course in Machine Design at the Indian Institute of Technology, Madras. His interests include buckling of columns, development of mechanical packages for electronic subsystems and stationary plasma thrusters. He is a co-author of internationally published papers on the use of strain gauges for ion thrust measurement.

K. G. Kalyan Kumar (Non-member) has obtained his

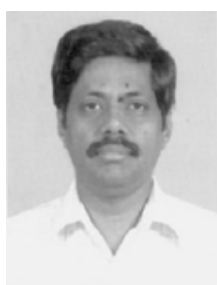

Bachelors Degree in science and a postoraduate Diploma in Instrumentation from Bangalore University during 1975. Subsequently, he obtained Masters Degree in Physics from Annamalai University during 1985 . He joined the Indian Space Research Organization (ISRO) during 1978. As a Deputy Manager at the Environmental Test Facility, ISRO, he looked after the operation, maintenance of environmental test chambers apart from building new facilities for special needs. Presently he is with control systems group for the realization of spacecraft electric propulsion system and creation of associated test set-up.
S. Nagabushanam (Non-member) has obtained his Mas-

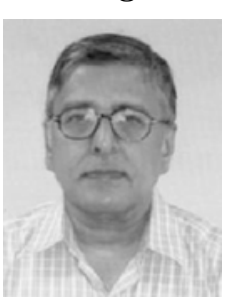
ters Degree in Machine Design from the Indian Institute of Technology, Madras during 1972 and has been with Indian Space Research organization (ISRO) Satellite Centre since 1973. As head of Control Components Division he looks after all the mechanical aspects regarding spacecraft control systems hardware, testing and simulation. His areas of interest include dry lubrication, magnetic suspension, magnetic torquer, conventional and electric propulsion. 